\title{
EDITORIAL
}

\section{Paul Ehrlich: founder of chemotherapy}

This year marks the 100th anniversary of the award of the Nobel Prize for Physiology or Medicine to Paul Ehrlich, in recognition of his contribution to immunology. His impact, however, extended far beyond this - for example, in his pioneering research in discovering new anti-infectives, which are once again in urgent demand.

A century ago, syphilis was a major public health threat in Europe. But by the 1920s, this situation was improved dramatically by the successful introduction of the first antibacterial drugs to combat the disease. These therapies resulted from the research of Paul Ehrlich (Supplementary information S1 (box)), who also received the Nobel Prize (with Elie Metchnikoff) 100 years ago in recognition of his work on immunity. In view of this centennial, and the current stagnation in antibacterial pipelines, it seems timely to reflect on the ground-breaking contributions of Ehrlich to the birth of chemotherapy (see REF. 1 for further reading).

Ehrlich's research career began with selective cell staining with dyes, which allowed him to identify mast cells and the different types of granulocytes. Such studies led him to formulate the concept of molecules that specifically bind to cell receptors; like a key that can only open the lock it was made for. In Ehrlich's beloved Latin terminology: Corpora non agunt nisi fixata (agents only work when they are bound). The key-lock principle led to the side-chain theory of antibody formation, which became a cornerstone of the newly emerging discipline of immunology, as recognized by the Nobel Prize.

Then, Ehrlich decided to revisit small molecules, now with the aim of finding a magic bullet or therapia sterilisans magna (great sterilizing therapy) to kill microbial pathogens. During his work with dyes, Ehrlich had tested the effects of methylene blue on malaria plasmodia, and so he first searched for drugs against parasites. Together with his postdoc, Shiga, he chose African trypanosomes as a target and trypan red as the drug, and soon established proof of principle in 1904. He also noted the first example of the emergence of resistance to chemotherapy.

Ehrlich next turned his attention to atoxyl, an arsenic compound that had already been used for treatment of sleeping sickness in Africa, which unfortunately had blindness as an intolerable side effect. Ehrlich, however, recognized the potential of chemical modifications of atoxyl to improve its safety.

Schaudinn and Hoffmann had identified the aetiological agent of syphilis, now known as Treponema pallidum, in 1905, and, importantly, Uhlenhuth and Salmon had earlier reported that atoxyl is not only active against trypanosomes, but also against fowl spirochetes. Ehrlich guided expert chemists at the Georg-Speyer-Haus Institute in Frankfurt to synthesize atoxyl derivatives for testing in a high-throughput system for screening for both antimicrobial and undesirable side effects at the adjacent Institute for Experimental Therapy, which he also headed (Supplementary information S2 (box)). Screening of derivatives up to the 606th preparation was done in mice infected with trypanosomes, and as this screen revealed high activity, compound 606 was tested against fowl spirochetes. It provided a rapid cure, and so Ehrlich turned to his ultimate target: T. pallidum.

Shortly before the discovery of compound 606, Uhlenhuth had adapted T. pallidum to provide a rabbit model, in which compound 606 turned out to be highly efficacious. The first clinical trial of 50 patients with late-stage syphilis using salvarsan (as Ehrlich had named compound 606) was conducted in 1909, and ended with an impressive positive outcome.

However, as salvarsan became more widely used, side effects became more frequently reported, and Ehrlich suffered a period of humiliating accusations. Undeterred, he analysed the origin of the side effects and found that the drug was rapidly degraded to toxic products when dissolved in impure water without basification or when the therapeutic solution was allowed to stand in air. To address these shortcomings, Ehrlich directed the chemical modification of salvarsan to produce a water-soluble analogue that did not require basification, yet retained its high therapeutic activity. After another 300 derivatives had been made, in 1912, compound 914 (neosalvarsan) proved to be safe and active - the mission was accomplished.

Ehrlich was fortunate to have at his disposal an institute with top chemists and an institute equipped for highthroughput animal testing. Moreover, a close relationship with the Hoechst Company existed, which immediately licensed the patents of the drugs and produced them in large quantities for clinical use. Thus, the translation of his research into a drug was rapidly achieved. Such public-private partnerships could help to accelerate antiinfective drug development today, an urgent need if we are to avoid the risk of falling back into the pre-antibiotic times of Ehrlich.

Stefan H. E. Kaufmann

1. Ehrlich, P. Chemotherapy (Pergamon Press, London, 1960). 